\title{
Cotype and absolutely summing homogeneous polynomials in $\mathcal{L}_{p}$ spaces
}

\author{
by \\ Daniel Pellegrino (Campina Grande)
}

\begin{abstract}
We lift to homogeneous polynomials and multilinear mappings a linear result due to Lindenstrauss and Pełczyński for absolutely summing operators. We explore the notion of cotype to obtain stronger results and provide various examples of situations in which the space of absolutely summing homogeneous polynomials is different from the whole space of homogeneous polynomials. Among other consequences, these results enable us to obtain answers to some open questions about absolutely summing homogeneous polynomials and multilinear mappings on $\mathcal{L}_{\infty}$ spaces.
\end{abstract}

1. Introduction and background. In the seminal paper [6], Lindenstrauss and Pełczyński provide a beautiful theorem which states that if $X$ is an infinite-dimensional Banach space with unconditional Schauder basis and every linear mapping from $X$ into an infinite-dimensional Banach space $Y$ is absolutely $(1 ; 1)$-summing, then $X$ is isomorphic to $l_{1}(\Gamma)$ and $Y$ is isomorphic to a Hilbert space. We will refine this statement by exploring the cotype of $Y$, not only for the linear cases, but also and mainly for homogeneous polynomials and multilinear mappings. As corollaries we obtain several negative results, showing, in particular, that various known Coincidence Theorems for polynomials and multilinear mappings cannot be improved in many natural ways.

Throughout this paper $X_{1}, \ldots, X_{n}, X, Y$ will stand for Banach spaces. The scalar field $\mathbb{K}$ can be either $\mathbb{R}$ or $\mathbb{C}$.

The Banach spaces of all continuous $n$-linear mappings from $X_{1} \times \ldots \times X_{n}$ into $Y$ endowed with the sup norm will be denoted by $\mathcal{L}\left(X_{1}, \ldots, X_{n} ; Y\right)$. For the Banach space of all continuous $n$-homogeneous polynomials $P$ from $X$ into $Y$ with the sup norm we use the symbol $\mathcal{P}\left({ }^{n} X, Y\right)$.

For the natural isometry

$$
\Psi_{t}: \mathcal{L}\left(X_{1}, \ldots, X_{n} ; Y\right) \rightarrow \mathcal{L}\left(X_{1}, \ldots, X_{t} ; \mathcal{L}\left(X_{t+1}, \ldots, X_{n} ; Y\right)\right)
$$

we write $\Psi_{t}(T)=T_{1}$.

2000 Mathematics Subject Classification: Primary 46B15; Secondary 46G25. 
The linear space of all sequences $\left(x_{j}\right)_{j=1}^{\infty}$ in $X$ such that

$$
\left\|\left(x_{j}\right)_{j=1}^{\infty}\right\|_{p}=\left(\sum_{j=1}^{\infty}\left\|x_{j}\right\|^{p}\right)^{1 / p}<\infty
$$

will be denoted by $l_{p}(X)$. We will also denote by $l_{p}^{w}(X)$ the linear space composed by the sequences $\left(x_{j}\right)_{j=1}^{\infty}$ in $X$ such that $\left(\left\langle\varphi, x_{j}\right\rangle\right)_{j=1}^{\infty} \in l_{p}(\mathbb{K})$ for every continuous linear functional $\varphi: X \rightarrow \mathbb{K}$. We define $\|\cdot\|_{w, p}$ in $l_{p}^{w}(X)$ by

$$
\left\|\left(x_{j}\right)_{j=1}^{\infty}\right\|_{w, p}=\sup _{\varphi \in B_{X^{\prime}}}\left(\sum_{j=1}^{\infty}\left|\left\langle\varphi, x_{j}\right\rangle\right|^{p}\right)^{1 / p} .
$$

The case $p=\infty$ is the case of bounded sequences and in $l_{\infty}(X)$ we use the sup norm. One can see that $\|\cdot\|_{p}$ (resp. $\|\cdot\|_{w, p}$ ) is a $p$-norm in $l_{p}(X)$ (resp. $\left.l_{p}^{w}(X)\right)$ for $p<1$, and a norm for $p \geq 1$. In any case, they are complete metrizable linear spaces.

Recall that if $2 \leq q \leq \infty$ and $\left(r_{j}\right)_{j=1}^{\infty}$ are the Rademacher functions, then $X$ has cotype $q$ if there exists $C_{q}(X) \geq 0$ such that, no matter how we choose $k \in \mathbb{N}$ and $x_{1}, \ldots, x_{k} \in X$,

$$
\left(\sum_{j=1}^{k}\left\|x_{j}\right\|^{q}\right)^{1 / q} \leq C_{q}(X)\left(\int_{0}^{1}\left\|\sum_{j=1}^{k} r_{j}(t) x_{j}\right\|^{2} d t\right)^{1 / 2} .
$$

To cover the case $q=\infty$ we replace $\left(\sum_{j=1}^{k}\left\|x_{j}\right\|^{q}\right)^{1 / q}$ by $\max _{j \leq n}\left\|x_{j}\right\|$. We will define the smallest cotype of $X$ by $\cot X$.

The theory of absolutely summing multilinear mappings was sketched by A. Pietsch in 1983 [12] and it was rapidly developed thereafter. The definitions of absolutely summing homogeneous polynomials and multilinear mappings we will work with in this paper were first explored by Alencar and Matos [1] and have been broadly used (see [2], [5], [11]).

Definition 1 (Alencar-Matos). A continuous multilinear mapping $T$ : $X_{1} \times \ldots \times X_{n} \rightarrow Y$ is absolutely $\left(p ; q_{1}, \ldots, q_{n}\right)$-summing (or $\left(p ; q_{1}, \ldots, q_{n}\right)$ summing $)$ if $\left(T\left(x_{j}^{(1)}, \ldots, x_{j}^{(n)}\right)\right)_{j=1}^{\infty} \in l_{p}(Y)$ for all $\left(x_{j}^{(s)}\right)_{j=1}^{\infty} \in l_{q_{s}}^{w}\left(X_{s}\right), s=$ $1, \ldots, n$. A continuous $n$-homogeneous polynomial $P: X \rightarrow Y$ is absolutely $(p ; q)$-summing (or $(p ; q)$-summing) if $\left(P\left(x_{j}\right)\right)_{j=1}^{\infty} \in l_{p}(Y)$ for all $\left(x_{j}\right)_{j=1}^{\infty} \in$ $l_{q}^{w}(X)$.

In order to avoid trivialities we assume that $p \geq q / n$ in the polynomial case and $1 / p \leq 1 / q_{1}+\ldots+1 / q_{n}$ in the $n$-linear case. We denote the space of absolutely $\left(p ; q_{1}, \ldots, q_{n}\right)$-summing $n$-linear mappings from $X_{1} \times \ldots \times X_{n}$ into $Y$ by $\mathcal{L}_{\text {as }\left(p ; q_{1}, \ldots, q_{n}\right)}\left(X_{1}, \ldots, X_{n} ; Y\right)$. When $q_{1}=\ldots=q_{n}=q$, we write $\mathcal{L}_{\text {as }(p ; q)}\left(X_{1}, \ldots, X_{n} ; Y\right)$. Analogously, the space of all $n$-homogeneous $(p ; q)$ summing polynomials from $X$ into $Y$ is denoted by $\mathcal{P}_{\text {as }(p ; q)}\left({ }^{n} X ; Y\right)$. 
In $\mathcal{L}_{\text {as }(p / n ; p)}\left(X_{1}, \ldots, X_{n} ; Y\right)$ and $\mathcal{P}_{\text {as }(p / n ; p)}\left({ }^{n} X ; Y\right)$ there is a natural version of the Grothendieck-Pietsch Domination Theorem and therefore the $(p / n ; p)$-summing $n$-homogeneous polynomials (resp. $n$-linear mappings) are called $p$-dominated polynomials (resp. $n$-linear mappings), as in Matos [7] and Tonge-Meléndez [9].

As in the linear case, we have a characterization theorem which plays a prominent role in the theory.

Theorem 1 (Matos [7]). Let $P$ be an $m$-homogeneous polynomial from $X$ into $Y$. Then the following statements are equivalent:

(1) $P$ is absolutely $(p ; q)$-summing.

(2) There exists $L>0$ such that

$$
\left(\sum_{j=1}^{k}\left\|P\left(x_{j}\right)\right\|^{p}\right)^{1 / p} \leq L\left\|\left(x_{j}\right)_{j=1}^{k}\right\|_{w, q}^{m} \quad \forall k \in \mathbb{N} \text { and } x_{j} \in X .
$$

(3) There exists $L>0$ such that

$$
\left(\sum_{j=1}^{\infty}\left\|P\left(x_{j}\right)\right\|^{p}\right)^{1 / p} \leq L\left\|\left(x_{j}\right)_{j=1}^{\infty}\right\|_{w, q}^{m} \quad \forall\left(x_{j}\right)_{j=1}^{\infty} \in l_{q}^{w}(X) .
$$

The infimum of the $L>0$ for which (1.1) holds is a norm for the case $p \geq 1$ and a $p$-norm for $p<1$ ([7]) on the space of $(p ; q)$-summing homogeneous polynomials. In any case, we thus obtain complete topological metrizable spaces. This norm (and $p$-norm) will be denoted by $\|\cdot\|_{\text {as }(p ; q)}$.

The characterization for the multilinear case and the definition of the norm (and $p$-norm) follow the same pattern.

The next theorem states the definitive crucial connection of absolutely summing linear mappings and cotype.

Theorem 2 (Maurey-Talagrand). If a Banach space $X$ has cotype $q$ then id $: X \rightarrow X$ is $(q ; 1)$-summing. The converse is true, except for $q=2$.

As a consequence of Theorem 2 and the Generalized Hölder Inequality one can prove the following result:

Theorem 3 (Botelho [2]). If $Y$ has cotype $q$, then

$$
\mathcal{L}\left({ }^{n} X ; Y\right)=\mathcal{L}_{\mathrm{as}(q ; 1)}\left({ }^{n} X ; Y\right) \quad \text { for all Banach spaces } X .
$$

If $X$ has cotype $q$, then

$$
\mathcal{L}\left({ }^{n} X ; Y\right)=\mathcal{L}_{\text {as }(q / n ; 1)}\left({ }^{n} X ; Y\right) \quad \text { for all Banach spaces } Y .
$$

In the next sections, among other results, we will prove that, in general, we cannot expect a result stronger than Theorem 3 . 
2. Main results. The remarkable works of Maurey-Pisier [8] and Lindenstrauss-Pełczyński [6] will play a fundamental role in this paper. We start with the following theorem, whose proof has inspired our results.

Theorem 4 (Lindenstrauss-Pełczyński [6, Theorem 4.2]). If $X$ has an unconditional Schauder basis, $\operatorname{dim} X=\operatorname{dim} Y=\infty$ and every bounded linear operator from $X$ into $Y$ is absolutely $(1 ; 1)$-summing, then $X$ is isomorphic to $l_{1}(\Gamma)$ and $Y$ is a Hilbert space.

This result and the Multilinear Grothendieck-Pietsch Domination Theorem lead us to interesting, although restricted, initial results.

ExAmple 1. Adapting an idea of M. C. Matos one can prove, for instance, that if $X$ has an unconditional Schauder basis, then

$$
\mathcal{L}\left({ }^{n} X ; \mathbb{K}\right) \neq \mathcal{L}_{\text {as }(1 / n ; 1)}\left({ }^{n} X ; \mathbb{K}\right)
$$

and thus,

$$
\mathcal{L}\left({ }^{n} X ; Y\right) \neq \mathcal{L}_{\text {as }(1 / n ; 1)}\left({ }^{n} X ; Y\right) \quad \text { for every Banach space } Y .
$$

Indeed, if we had $\mathcal{L}\left({ }^{2} X ; \mathbb{K}\right)=\mathcal{L}_{\text {as }(1 / 2 ; 1)}\left({ }^{2} X ; \mathbb{K}\right)$, then given $S: X \rightarrow X^{\prime}$, we could define $T_{S}: X \times X \rightarrow \mathbb{K}$ such that $\left(T_{S}\right)_{1}=S$. By hypothesis, $T_{S}$ would be $(1 / 2 ; 1,1)$-summing. Hence, by the Grothendieck-Pietsch Domination Theorem, there exists $C>0$ such that

$$
\left\|T_{S}(x, y)\right\| \leq C\left(\int_{B_{X^{\prime}}}|\varphi(x)| d \mu_{1}\right)\left(\int_{B_{X^{\prime}}}|\psi(y)| d \mu_{2}\right)
$$

and

$$
\left\|\left(T_{S}\right)_{1}(x)\right\|=\sup _{\|y\| \leq 1}\left\|T_{S}(x, y)\right\| \leq C \int_{B_{X^{\prime}}}|\varphi(x)| d \mu_{1} .
$$

Then $\|S(x)\| \leq C \int_{B_{X^{\prime}}}|\varphi(x)| d \mu_{1}$ and hence $\mathcal{L}\left(X ; X^{\prime}\right)=\mathcal{L}_{\text {as }(1 ; 1)}\left(X ; X^{\prime}\right)$ (contradiction by Theorem 4 ). The general case follows by a standard inductive process.

The same simple construction gives many other results. However, the previous negative results, albeit interesting, are confined to the dominated cases (which have Grothendieck-Pietsch Domination Theorem as a fundamental tool) and do not give us the full picture. In general, the spaces of $p$-dominated homogeneous polynomials and multilinear mappings are small, and negative results are not surprising. We will present new negative results which significantly improve the last ones. Our approach consists in lifting Theorem 4 to polynomial and multilinear versions and refining them by exploring the properties of cotype.

Assume that $X$ and $Y$ are two infinite-dimensional Banach spaces, $X$ with unconditional Schauder basis $\left(x_{n}\right)$ with coefficient functionals $\left(x_{n}^{*}\right)$, 
and moreover $\mathcal{P}_{\operatorname{as}(q ; 1)}\left({ }^{m} X ; Y\right)=\mathcal{P}\left({ }^{m} X ; Y\right)$ with $1 / m \leq q$. We will deal with the following general question:

What is the best (smallest) $t, 1 \leq t \leq \infty$, such that in this situation $\left(x_{n}^{*}(x)\right) \in l_{t}$ for each $x \in X$ ? Henceforth the best such $t$ will be denoted by $\mu=\mu(X, Y, q, m)$.

Firstly let us recall a well known concept:

Definition 2. We say that $Y$ finitely factors (ff) the formal inclusion $l_{p} \rightarrow l_{\infty}$ for $0<\delta<1$ if for every $n$ there exist $y_{1}, \ldots, y_{n}$ such that

$$
(1-\delta)\|a\|_{\infty} \leq\left\|\sum_{k \leq n} a_{k} y_{k}\right\| \leq\|a\|_{p} \quad \text { for all } a=\left(a_{k}\right)_{k=1}^{n} \in l_{p}^{n} .
$$

Note that $(1-\delta)\left|a_{k}\right| \leq\left\|a_{k} y_{k}\right\| \leq\left|a_{k}\right|$ and then $1-\delta \leq\left\|y_{k}\right\| \leq 1$ for all $k$.

TheOrem 5. Let $X$ and $Y$ be infinite-dimensional Banach spaces. Suppose that $X$ has an unconditional Schauder basis $\left(x_{n}\right)$. If $Y$ finitely factors the formal inclusion $l_{p} \rightarrow l_{\infty}$ for some $\delta$ and $\mathcal{P}_{\text {as }(q ; 1)}\left({ }^{m} X ; Y\right)=\mathcal{P}\left({ }^{m} X ; Y\right)$ with $1 / m \leq q$, then

(a) $\mu \leq m p q /(p-q)$ if $q<p$,

(b) $\mu \leq m q$ if $q \leq p / 2$.

Proof. We can suppose, with no loss of generality, that $\left(x_{n}\right)$ is normalized. Consider $q \in[1 / m, p[$. By hypothesis, there exists $K>0$ such that $\|P\|_{\text {as }(q ; 1)} \leq K\|P\|$ for all $P \in \mathcal{P}\left({ }^{m} X ; Y\right)$. by

Let $\left\{\mu_{i}\right\}_{i=1}^{n}$ be such that $\sum_{j=1}^{n}\left|\mu_{j}\right|^{s}=1$ with $s=p / q$. Define $P: X \rightarrow Y$

$$
P x=\sum_{j=1}^{n}\left|\mu_{j}\right|^{1 / q} a_{j}^{m} y_{j} \quad \text { if } x=\sum_{j=1}^{\infty} a_{j} x_{j},
$$

where the $y_{j}$ satisfy $(1-\delta)\|a\|_{\infty} \leq\left\|\sum_{k \leq n} a_{k} y_{k}\right\| \leq\|a\|_{p}$ for all $a=$ $\left(a_{k}\right)_{k=1}^{n} \in l_{p}^{n}$. Since $\left(x_{n}\right)$ is an unconditional basis, there exists $\varrho>0$ satisfying

$$
\left\|\sum_{j=1}^{\infty} \varepsilon_{j} a_{j} x_{j}\right\| \leq \varrho\left\|\sum_{j=1}^{\infty} a_{j} x_{j}\right\|=\varrho\|x\| \quad \text { for any } \varepsilon_{j}=1 \text { or } \varepsilon_{j}=-1 .
$$

Hence $\left\|\sum_{j=1}^{n} \varepsilon_{j} a_{j} x_{j}\right\| \leq \varrho\|x\|$ for all $n$ and any $\varepsilon_{j}=1$ or -1 and then we have

$$
\begin{aligned}
\|P x\| & =\left\|\sum_{j=1}^{n}\left|\mu_{j}\right|^{1 / q} a_{j}^{m} y_{j}\right\| \leq\left(\left.\left.\sum_{j=1}^{n}|| \mu_{j}\right|^{1 / q} a_{j}^{m}\right|^{p}\right)^{1 / p} \\
& \leq \varrho^{m}\|x\|^{m}\left(\sum_{j=1}^{n}\left|\mu_{j}\right|^{p / q}\right)^{1 / p}=\varrho^{m}\|x\|^{m}\left(\sum_{j=1}^{n}\left|\mu_{j}\right|^{s}\right)^{1 / p}=\varrho^{m}\|x\|^{m} .
\end{aligned}
$$


We obtain $\|P\| \leq \varrho^{m}$ and $\|P\|_{\text {as }(q ; 1)} \leq K \varrho^{m}$ and achieve the estimate below:

$$
\begin{aligned}
\left(\left.\left.\sum_{j=1}^{n}\left|a_{j}^{m}\right| \mu_{j}\right|^{1 / q}(1-\delta)\right|^{q}\right)^{1 / q} & \leq\left(\sum_{j=1}^{n}\left\|a_{j}^{m}\left|\mu_{j}\right|^{1 / q} y_{j}\right\|^{q}\right)^{1 / q} \\
& =\left(\sum_{j=1}^{n}\left\|P a_{j} x_{j}\right\|^{q}\right)^{1 / q} \\
& \leq\|P\|_{\operatorname{as}(q ; 1)}\left\|\left(a_{j} x_{j}\right)_{j=1}^{n}\right\|_{w, 1}^{m} \\
& \leq\|P\|_{\operatorname{as}(q ; 1)} \max _{\varepsilon_{j} \in\{1,-1\}}\left\{\left\|\sum_{j=1}^{n} \varepsilon_{j} a_{j} x_{j}\right\|\right\}^{m} \\
& \leq\|P\|_{\operatorname{as}(q ; 1)}(\varrho\|x\|)^{m} \leq K \varrho^{2 m}\|x\|^{m} .
\end{aligned}
$$

Since $\frac{1}{s}+\frac{1}{\frac{s}{s-1}}=1$ we have

$$
\begin{aligned}
\left(\sum_{j=1}^{n}\left|a_{j}\right|^{\frac{s}{s-1} m q}\right)^{1 / \frac{s}{s-1}} & =\left\|\left(\left|a_{j}\right|^{m q}\right)_{j=1}^{n}\right\|_{\frac{s}{s-1}} \\
& =\sup \left\{\left.\left.\left|\sum_{j=1}^{n} \mu_{j}\right| a_{j}\right|^{m q}\left|: \sum_{j=1}^{n}\right| \mu_{j}\right|^{s}=1\right\} \\
& \leq \sup \left\{\sum_{j=1}^{n}\left|\mu_{j}\right|\left|a_{j}\right|^{m q}: \sum_{j=1}^{n}\left|\mu_{j}\right|^{s}=1\right\}
\end{aligned}
$$

It is plain that (2.1) holds whenever $\sum_{j=1}^{n}\left|\mu_{j}\right|^{s}=1$. Thus, by (2.1) and (2.2) it follows that

$$
\left(\sum_{j=1}^{n}\left|a_{j}\right|^{\frac{s}{s-1} m q}\right)^{1 / \frac{s}{s-1}} \leq\left[(1-\delta)^{-1} K \varrho^{2 m}\|x\|^{m}\right]^{q}
$$

and then

$$
\left(\sum_{j=1}^{n}\left|a_{j}\right|^{\frac{s}{s-1} m q}\right)^{1 / \frac{s}{s-1} m q} \leq\left[(1-\delta)^{-1} K \varrho^{2 m}\|x\|^{m}\right]^{1 / m} .
$$

Since $\frac{s}{s-1} m q=\frac{m p q}{p-q}$ and $n$ is arbitrary, part (a) is proved. Now, if $1 / m \leq$ $q \leq p / 2$, define $S: X \rightarrow Y$ by

$$
S x=\sum_{j=1}^{n} a_{j}^{m} y_{j} \quad \text { if } x=\sum_{j=1}^{\infty} a_{j} x_{j} .
$$

Since $m p \geq \frac{s}{s-1} m q$, we obtain 


$$
\begin{aligned}
\|S x\| & =\left\|\sum_{j=1}^{n} a_{j}^{m} y_{j}\right\| \leq\left(\sum_{j=1}^{n}\left|a_{j}^{m}\right|^{p}\right)^{1 / p}=\left[\left(\sum_{j=1}^{n}\left|a_{j}\right|^{m p}\right)^{1 / m p}\right]^{m} \\
& \leq\left[\left(\sum_{j=1}^{n}\left|a_{j}\right|^{\frac{s}{s-1} m q}\right)^{1 / \frac{s}{s-1} m q}\right]^{m} \leq(1-\delta)^{-1} K \varrho^{2 m}\|x\|^{m} .
\end{aligned}
$$

Thus $\|S\| \leq(1-\delta)^{-1} K \varrho^{2 m}$ and $\|S\|_{\text {as }(q ; 1)} \leq(1-\delta)^{-1} K^{2} \varrho^{2 m}$. Hence

$$
\begin{aligned}
& \sum_{j=1}^{n}\left|a_{j}^{m}(1-\delta)\right|^{q} \leq \sum_{j=1}^{n}\left\|a_{j}^{m} y_{j}\right\|^{q}=\sum_{j=1}^{n}\left\|S a_{j} x_{j}\right\|^{q} \\
& \quad \leq\|S\|_{\mathrm{as}(q ; 1)}^{q} \max _{\varepsilon_{j} \in\{1,-1\}}\left\{\left\|\sum_{j=1}^{n} \varepsilon_{j} a_{j} x_{j}\right\|\right\}^{m q} \leq\left((1-\delta)^{-1} K^{2} \varrho^{2 m}\right)^{q}(\varrho\|x\|)^{m q} .
\end{aligned}
$$

Consequently, since $n$ is arbitrary, we have $\sum_{j=1}^{\infty}\left|a_{j}\right|^{m q}<\infty$ whenever $x=\sum_{j=1}^{\infty} a_{j} x_{j} \in X$.

The well known Dvoretzky-Rogers Theorem asserts that every infinitedimensional Banach space finitely factors the formal inclusion $l_{2} \rightarrow l_{\infty}$. So we have the following result:

Corollary 1. Let $X$ and $Y$ be infinite-dimensional Banach spaces. Suppose that $X$ has an unconditional Schauder basis. If $\mathcal{P}_{\operatorname{as}(q ; 1)}\left({ }^{m} X ; Y\right)=$ $\mathcal{P}\left({ }^{m} X ; Y\right)$ with $1 / m \leq q$, then

(a) $\mu \leq 2 m q /(2-q)$ if $q<2$,

(b) $\mu \leq m q$ if $q \leq 1$.

The following characterization of cotype is crucial to obtain important improvements of Corollary 1.

TheOREM 6 (Maurey-Pisier [8]). For any infinite-dimensional Banach space $Y$ we have

$$
\inf \{2 \leq p \leq \infty: Y \text { has cotype } p\}=\sup \left\{2 \leq p \leq \infty: Y \text { ff } l_{p} \rightarrow l_{\infty}\right\} .
$$

Now, Theorems 5 and 6 have the following consequences:

Corollary 2. Let $X$ and $Y$ be infinite-dimensional Banach spaces. Suppose that $X$ has an unconditional Schauder basis and $\cot Y<\infty$. If $\mathcal{P}_{\text {as }(q ; 1)}\left({ }^{m} X ; Y\right)=\mathcal{P}\left({ }^{m} X ; Y\right)$ with $1 / m \leq q$, then

(a) $\mu \leq m q \cot Y /(\cot Y-q)$ if $q<\cot Y$,

(b) $\mu \leq m q$ if $q \leq(\cot Y) / 2$.

The next three corollaries deal with the converse of the polynomial version of Theorem 3.

Corollary 3. If $Y$ is an infinite-dimensional Banach space, $\cot Y<\infty$, $q \in[1 / m,(\cot Y) / 2]$ and $\mathcal{P}\left({ }^{m} l_{t} ; Y\right)=\mathcal{P}_{\text {as }(q ; 1)}\left({ }^{m} l_{t} ; Y\right)$ then $t \leq m q$. 
Corollary 4. If $Y$ is an infinite-dimensional Banach space and $\cot Y$ $<\infty$ then

$$
\mathcal{P}\left({ }^{m} c_{0} ; Y\right)=\mathcal{P}_{\text {as }(q ; 1)}\left({ }^{m} c_{0} ; Y\right) \Leftrightarrow q \geq \cot Y .
$$

Obviously localization allows one to replace $c_{0}$ by any $\mathcal{L}_{\infty}$ space in Corollary 4.

Corollary 5. If $\mathcal{P}\left({ }^{m} l_{t} ; l_{p}\right)=\mathcal{P}_{\text {as }(q ; 1)}\left({ }^{m} l_{t} ; l_{p}\right)$ for $q \in\left[1 / m\right.$, $\cot l_{p}[$, then

$$
t \leq \frac{m q \max \{p, 2\}}{\max \{p, 2\}-q}
$$

The case that $Y$ has only infinite cotype can also be obtained as a consequence of Theorem 5. Precisely, we have:

Theorem 7. Let $X$ and $Y$ be infinite-dimensional Banach spaces. Suppose that $X$ has an unconditional Schauder basis and $\cot Y=\infty$. If

$$
\mathcal{P}_{\text {as }(q ; 1)}\left({ }^{m} X ; Y\right)=\mathcal{P}\left({ }^{m} X ; Y\right)
$$

with $1 / m \leq q$, then $\mu \leq m q$.

Proof. Since $\cot Y=\infty$, Theorem 6 asserts that $Y$ finitely factors the formal inclusion $l_{p} \rightarrow l_{\infty}$ for every $p \geq 2$. Thus, by Theorem 5 we have $\mu \leq \lim _{p \rightarrow \infty} m p q /(p-q)=m q$.

Now, a standard localization argument gives the following straightforward consequence:

Corollary 6. If $\cot Y=\infty$ and $X$ is an $\mathcal{L}_{\infty}$ space, then

$$
\mathcal{P}\left({ }^{m} X ; Y\right) \neq \mathcal{P}_{\text {as }(r ; s)}\left({ }^{m} X ; Y\right) \quad \text { for every } r>0 \text { and } s \geq 1 .
$$

It is plain that whenever $\mathcal{P}\left({ }^{n} X ; Y\right) \neq \mathcal{P}_{\text {as }(r ; s)}\left({ }^{n} X ; Y\right)$ we also obtain $\mathcal{L}\left({ }^{n} X ; Y\right) \neq \mathcal{L}_{\text {as }(r ; s)}\left({ }^{n} X ; Y\right)$. Moreover, we can consider the following problem:

If $X_{1}, \ldots, X_{m}$ and $Y$ are infinite-dimensional Banach spaces, each $X_{j}$ has an unconditional Schauder basis $\left(x_{j, n}\right)$ with coefficient functionals $\left(x_{j, n}^{*}\right)$ and moreover $\mathcal{L}_{\text {as }(q ; 1)}\left(X_{1}, \ldots, X_{m} ; Y\right)=\mathcal{L}\left(X_{1}, \ldots, X_{m} ; Y\right)$ with $1 / m \leq q$, what is the best (smallest) $t, 1 \leq t \leq \infty$, such that $\left(\prod_{j=1}^{m} x_{j, n}^{*}\left(x_{j}\right)\right) \in l_{t}$ for every $x_{j} \in X_{j}, j=1, \ldots, m ?$

Denoting the best such $t$ by $\mu=\mu\left(X_{1}, \ldots, X_{m}, Y, q, m\right)$, one can realize that the polynomial proof can be adjusted to give the following result:

Theorem 8. Let $Y$ be an infinite-dimensional Banach space and let $X_{1}, \ldots, X_{m}$ denote infinite-dimensional Banach spaces with unconditional Schauder basis. If $Y$ finitely factors the formal inclusion $l_{p} \rightarrow l_{\infty}$ for some $\delta$ and $\mathcal{L}_{\mathrm{as}(q ; 1)}\left(X_{1}, \ldots, X_{m} ; Y\right)=\mathcal{L}\left(X_{1}, \ldots, X_{m} ; Y\right)$ with $1 / m \leq q$, then

(a) $\mu \leq p q /(p-q)$ if $q<p$,

(b) $\mu \leq q$ if $q \leq p / 2$. 
3. Final applications. The following simple result, whose proof we will omit, added to our negative results will provide some simple answers to interesting open questions about absolutely summing polynomials and multilinear mappings.

Proposition 1. If

$$
\mathcal{L}\left(X_{1}, \ldots, X_{n} ; Y\right)=\mathcal{L}_{\mathrm{as}\left(r ; s_{1}, \ldots, s_{t}, \infty, \ldots, \infty\right)}\left(X_{1}, \ldots, X_{n} ; Y\right)
$$

then

$$
\begin{aligned}
\mathcal{L}\left(X_{1}, \ldots, X_{t} ; \mathcal{L}\left(X_{t+1}, \ldots, X_{n} ; Y\right)\right) & \\
& =\mathcal{L}_{\mathrm{as}\left(r ; s_{1}, \ldots, s_{t}\right)}\left(X_{1}, \ldots, X_{t} ; \mathcal{L}\left(X_{t+1}, \ldots, X_{n} ; Y\right)\right),
\end{aligned}
$$

and reciprocally.

Now, Theorem 8 and Proposition 1 yield the results below, which will be important at the end of the paper.

Proposition 2. Let $n \geq 3, r_{1}, \ldots, r_{n} \in[1, \infty]$ and $X_{1}, \ldots, X_{n}$ be infinite-dimensional $\mathcal{L}_{\infty}$ spaces such that

$$
\mathcal{L}\left(X_{1}, \ldots, X_{n} ; \mathbb{K}\right)=\mathcal{L}_{\text {as }\left(s ; r_{1}, \ldots, r_{n}\right)}\left(X_{1}, \ldots, X_{n} ; \mathbb{K}\right) .
$$

Then no more than one $r_{j}$ can be $\infty$.

Proof. We can assume $X_{1}=\ldots=X_{n}=c_{0}$. Suppose, with no loss of generality, that $r_{n-1}=r_{n}=\infty$. Hence, by Proposition 1 we would have

$$
\mathcal{L}\left(X_{1}, \ldots, X_{n-2} ; Z\right)=\mathcal{L}_{\mathrm{as}\left(s ; r_{1}, \ldots, r_{n-2}\right)}\left(X_{1}, \ldots, X_{n-2} ; Z\right)
$$

with $Z=\mathcal{L}\left(X_{n-1}, X_{n} ; \mathbb{K}\right)$. But $\cot \mathcal{L}\left(X_{n-1}, X_{n} ; \mathbb{K}\right)=\infty$ and (a corollary of) Theorem 8 asserts that this is impossible.

The same reasoning furnishes the next result.

Proposition 3. Let $n$ be a natural number, $n \geq 2, r_{1}, \ldots, r_{n} \in[1, \infty]$ and $\operatorname{dim} Y=\infty$. If $X_{1}, \ldots, X_{n}$ are infinite-dimensional $\mathcal{L}_{\infty}$ spaces and $\mathcal{L}\left({ }^{n} X ; Y\right)=\mathcal{L}_{\text {as }\left(s ; r_{1}, \ldots, r_{n}\right)}\left({ }^{n} X ; Y\right)$, then $r_{j} \neq \infty$ for all $j$.

Now we list two recent coincidence theorems; our previous results will show that they cannot be improved in some natural directions.

Theorem 9 (D. Pérez-García [11]). If $n \geq 2$ and each $X_{j}$ is an $\mathcal{L}_{\infty}$ space, then every continuous multilinear mapping from $X_{1} \times \ldots \times X_{n}$ into $\mathbb{K}$ is $(1 ; 2, \ldots, 2)$-summing.

It is clear that, in particular, Theorem 8 applied to $X_{1}, \ldots, X_{n}=c_{0}$ and a standard localization argument imply that Theorem 9 cannot be improved to an infinite-dimensional $Y$ in place of $\mathbb{K}$.

As a consequence of the last theorem, we have the following result. 
Theorem 10 (Pellegrino [10]). If $n \geq 2$ and each $X_{j}$ is an $\mathcal{L}_{\infty}$ space then

$$
\mathcal{L}\left(X_{1}, \ldots, X_{n} ; \mathbb{K}\right)=\mathcal{L}_{\text {as }(2 ; 2, \ldots, 2, \infty)}\left(X_{1}, \ldots, X_{n} ; \mathbb{K}\right) .
$$

Proof. Let $T: X_{1} \times \ldots \times X_{n} \rightarrow \mathbb{K}$ be a continuous $n$-linear mapping. Using Theorem 9 it is not hard to prove that every continuous multilinear mapping from $\mathcal{L}_{\infty}$ spaces into a cotype 2 space is $(2 ; 2, \ldots, 2)$-summing. Thus, since $\cot X_{n}^{\prime}=2$ we can assert that $T_{1}: X_{1} \times \ldots \times X_{n-1} \rightarrow X_{n}^{\prime}$ is $(2 ; 2, \ldots, 2)$-summing. Now, consider $\left(x_{j}^{(k)}\right)_{j=1}^{\infty} \in l_{2}^{w}\left(X_{k}\right), 1 \leq k \leq n-1$. Hence there exists $C>0$ such that

$$
\left(\sum_{j=1}^{\infty}\left\|T_{1}\left(x_{j}^{(1)}, \ldots, x_{j}^{(n-1)}\right)\right\|^{2}\right)^{1 / 2} \leq C \prod_{k=1}^{n-1}\left\|\left(x_{j}^{(k)}\right)_{j=1}^{\infty}\right\|_{w, 2} .
$$

If $\left(x_{j}^{(n)}\right)_{j=1}^{\infty} \in l_{\infty}\left(X_{n}\right)$ is non-zero, we obtain

$$
\left(\sum_{j=1}^{\infty}\left\|T_{1}\left(x_{j}^{(1)}, \ldots, x_{j}^{(n-1)}\right)\left(\frac{x_{j}^{(n)}}{\left\|\left(x_{j}^{(n)}\right)_{j=1}^{\infty}\right\|_{\infty}}\right)\right\|^{2}\right)^{1 / 2} \leq C \prod_{k=1}^{n-1}\left\|\left(x_{j}^{(k)}\right)_{j=1}^{\infty}\right\|_{w, 2} .
$$

Hence

$$
\left(\sum_{j=1}^{\infty}\left\|T\left(x_{j}^{(1)}, \ldots, x_{j}^{(n)}\right)\right\|^{2}\right)^{1 / 2} \leq C\left\|\left(x_{j}^{(n)}\right)_{j=1}^{\infty}\right\|_{\infty} \prod_{k=1}^{n-1}\left\|\left(x_{j}^{(k)}\right)_{j=1}^{\infty}\right\|_{w, 2} .
$$

The case $\left(x_{j}^{(n)}\right)_{j=1}^{\infty}=0$ does not cause any trouble.

The above result generalizes a bilinear result due to Botelho ([2] and [3]). In [3] it is asked whether

$$
\mathcal{L}\left({ }^{2} C(K) ; \mathbb{K}\right)=\mathcal{L}_{\text {as }(2 ; 2, \infty)}\left({ }^{2} C(K) ; \mathbb{K}\right)
$$

could be improved to some infinite-dimensional Banach space $Y$ in place of $\mathbb{K}$ or not.

In particular, Proposition 3 answers negatively this question and not only for the bilinear case, but for any $n$-linear case of (3.1).

Another question raised in [3] was:

Do we have $\mathcal{P}\left({ }^{n} C(K) ; Y\right) \neq \mathcal{P}_{\text {as }(r / n ; r)}\left({ }^{n} C(K) ; Y\right)$ for every $n>2, r<\infty$ and every Banach space $Y$ ?

If $\operatorname{dim} Y=\infty$, Corollary 1 gives a partial answer when $r / n<2$. But Corollary 4 achieves a more general result when $\cot Y<\infty$ since it asserts that whenever $r<n \cot Y$ we have $\mathcal{P}\left({ }^{n} C(K) ; Y\right) \neq \mathcal{P}_{\text {as }(r / n ; r)}\left({ }^{n} C(K) ; Y\right)$.

Finally, Corollary 6 shows that when $Y$ does not have finite cotype, we have $\mathcal{P}\left({ }^{n} C(K) ; Y\right) \neq \mathcal{P}_{\text {as }(r ; s)}\left({ }^{n} C(K) ; Y\right)$ for any $r>0$ and $s \geq 1$, which is a complete answer and goes beyond the dominated case. 
This paper forms a part of the author's doctoral thesis written at UNICAMP under the supervision of M. C. Matos. The author thanks Professor Matos, Professor J. Mujica and the referee for their suggestions.

\section{References}

[1] R. Alencar and M. C. Matos, Some classes of multilinear mappings between Banach spaces, Publ. Depto Anál. Mat., Univ. Complut. Madrid, Section 1, 12 (1989).

[2] G. Botelho, Cotype and absolutely summing multilinear mappings and homogeneous polynomials, Proc. Roy. Irish Acad. Sect. A 97 (1997), 145-153.

[3] -, Tipo e cotipo: Caracterização via funções de Rademacher generalizadas e contribuições à teoria de aplicações multilineares e polinômios homogêneos em espaços de Banach, doctoral thesis, UNICAMP, 1995.

[4] J. Diestel, H. Jarchow and A. Tonge, Absolutely Summing Operators, Cambridge Stud. Adv. Math. 43, Cambridge Univ. Press, Cambridge, 1995.

[5] K. Floret and M. C. Matos, Application of a Khinchine inequality to holomorphic mappings, Math. Nachr. 176 (1995), 65-72.

[6] J. Lindenstrauss and A. Pełczyński, Absolutely summing operators in $\mathcal{L}_{p}$ spaces and their applications, Studia Math. 29 (1968), 275-326.

[7] M. C. Matos, Absolutely summing holomorphic mappings, An. Acad. Brasil. Ciênc. 68 (1996), 1-13.

[8] B. Maurey et G. Pisier, Séries de variables aléatoires vectorielles indépendantes et proprietés géométriques des espaces de Banach, Studia Math. 58 (1976), 45-90.

[9] Y. Meléndez and A. Tonge, Polynomials and the Pietsch domination theorem, Proc. Roy. Irish Acad. Sect. A 99 (1999), 195-212.

[10] D. Pellegrino, Cotype and nonlinear absolutely summing mappings, preprint.

[11] D. Pérez-García, Operadores multilineales absolutamente sumantes, dissertation, Univ. Complut. Madrid, 2002.

[12] A. Pietsch, Ideals of multilinear functionals (designs of a theory), in: Proc. 2nd Internat. Conf. on Operator Algebras, Ideals, and their Applications in Theoretical Physics (Leipzig, 1983), Teubner-Texte Math. 67, Teubner, Leipzig, 1984, 185-199.

Departamento de Matemática e Estatística

Caixa Postal 10044

Campina Grande, PB, Brazil

Cep 58109-970

E-mail: dmp@dme.ufpb.br

Received April 30, 2002

Revised version October 21, 2002 